\title{
Comments on the significance and application sphere of SPR based fiber-optic sensors
}

\begin{abstract}
This opinion pertains to the significance of surface plasmon resonance based fiber-optic sensors for sensing applications in contemporary research and industrial avenues. The advantages of these sensors have been highlighted along with a few important examples.
\end{abstract}

\author{
Volume 6 Issue 2 - 2020
}

\author{
Rana Tabassum,' Ravi Kant ${ }^{2}$ \\ 'Centre for Nanoscience and Nanotechnology, Jamia Millia \\ Islamia (A Central University), India \\ ${ }^{2}$ Department of Physics, Indian Institute of Technology, India
}

Correspondence: Rana Tabassum, Centre for Nanoscience and Nanotechnology, Jamia Millia Islamia (A Central University), New Delhi-I I0025, India, Email rtabassum I@jmi.ac.in

Received: March II, 2020| Published: June 29, 2020

\section{Opinion}

Over past three decades, optical sensors based on surface plasmon resonance (SPR) as the underlying sensing principle have established themselves as analytically prominent and highly versatile sensing tools. ${ }^{1,2}$ The co-relation of the shift attained in the resonance parameter induced due to variation in refractive index of the sensing analyte forms the basis of an SPR based optical sensor which can be realized on different optical substrates, such as, prism, grating or optical fiber. ${ }^{3}$ Among these, an optical fiber projects maximum importance in the avenue of sensor technology because of myriad advantages and features which are immensely beneficial for sensing applications. In addition to exhibiting superior sensing performance demonstrated through high sensitivity, large calibration range, low detection limit and short response time, fiber-optic SPR sensors furnish various other advantages, such as, compactness, flexible and simple probe design and excellent prospects for miniaturization of sensing probe. ${ }^{4}$ The implementation of SPR on an optical fiber renders it immune towards electromagnetic interference, and therefore, fiberoptic SPR sensors can be safely used in hazardous areas. Moreover, their inherent compactness impart them with online monitoring and remote sensing feasibilities which are crucial from the perspective of large scale employment of these fiber-optic sensors at both laboratory and industrial scales. ${ }^{3,4}$ These advantages of SPR based fiber-optic sensors provide them an edge over various other sensing techniques, for instance, electrochemical, chromatography, fluorescence, potentiometry, among others. The use of nanostructured materials in synthesizing the sensing layer specific to a particular analyte broadens the application sphere of SPR based fiber-optic sensors by providing them high sensitivity and selectivity. ${ }^{5-8}$ In that aspect, various high refractive index metal oxides have been used to synthesize sensing layer to enhance the sensitivity of SPR based fiber-optic sensors. ${ }^{9,10}$ The employment of metal oxides having high refractive index has been carried out in the form of both bulk nanolayers as well as nanostructures of a variety of shapes. ${ }^{7,8,10}$

The mentioned advantages and attributes enable the fiber-optic SPR sensors to be used in a variety of sensing applications in diverse disciples. These include measurement of physical parameters, such as, refractive index, ${ }^{10,11}$ temperature and humidity in addition to gas sensing applications. ${ }^{4}$ The excellent specificity of SPR technique towards target analyte has been utilized to probe antigen-antibody interactions $^{2}$ and fabricate enzymatic fiber-optic biosensors. ${ }^{3,7,8}$ In addition, SPR technique has also been used to study reaction kinetics. ${ }^{2}$ The popularity of SPR technique in avenues other than sensing underscore its versatility in addition to emphasizing its novelty towards material characterization. Further, SPR based fiber-optic sensors are used for the sensing and quantification of numerous chemicals and biomolecules of commercial and biomedical significance. ${ }^{7,8,12,13}$ The importance of these sensors also stems from the fact that they facilitate multi-channel sensing significant from biological point of view. In that aspect, a cascaded channel fiber-optic SPR sensor has been reported for simultaneous detection of vitamin $\mathrm{K}_{1}$ and heparin such that vitamin $K_{1}$ is sensed on first channel to monitor blood coagulation while the second sensing channel is specific to heparin required for the therapy. ${ }^{14}$

\section{Conclusion}

In view of the indicated advantages, SPR based fiber-optic sensors hold great prospects for sensing applications in diverse fields of scientific research and industrial applications.

\section{Acknowledgements}

Rana Tabassum is thankful to INSPIRE faculty scheme of DST.

\section{Conflicts of interest}

The authors declare that there is no conflict of interest.

\section{References}

1. Abdulhalim I, Zourob M, Lakhtakia A. Surface plasmon resonance for biosensing-a mini review. Electromagnetics. 2008;28(3):213-242.

2. Couture M, Zhao SS, Masson JF. Modern surface plasmon resonance for bioanalytics and biophysics. PhysChemChemPhys. 2013;15:1119011216.

3. Gupta BD, Kant R. Recent advances in surface plasmon resonance based fiber optic chemical and biosensors utilizing bulk and nanostructures. OptLaser Technol. 2018;101:144-161.

4. Tabassum R, Kant R. Recent trends in surface plasmon resonance based fiber-optic gas sensors utilizing metal oxides and carbon nanomaterials as functional entities. SensActuators B. 2020;310:127813. 
5. Shobin LR, Sastikumar D, Manivannan S. Glycerol mediated synthesis of silver nanowires for room temperature ammonia vapor sensing. SensActuators A. 2014;21474-480.

6. He L, Pagneux Q, Larroulet I, et al. Label-free femtomolar cancer biomarker detection in human serum using graphene-coated surface plasmon resonance chips. BiosensBioelectron. 2017;89:606-611.

7. Kant R, Tabassum R, Gupta BD. Xanthine oxidase functionalized $\mathrm{Ta}_{2} \mathrm{O}_{5}$ nanostructures as a novel scaffold for highly sensitive SPR based fiber optic xanthine sensor. BiosensBioelectron. 2018;99:637-645.

8. Kant R, Gupta BD. Fiber-optic SPR based acetylcholine biosensor using enzyme functionalized $\mathrm{Ta}_{2} \mathrm{O}_{5}$ nanoflakes for Alzheimer's disease diagnosis.IEEE JLightwave Technol. 2018;36(18):4018-4024.

9. Shalabney A, Abdulhalim I. Electromagnetic fields distribution in multilayer thin film structures and the origin of sensitivity enhancement in surface plasmon resonance sensors.SensActuators A. 2010;159:24-32.

10. Tabassum R, Gupta BD. Influence of oxide overlayer on the performance of a fiber optic SPR sensor with $\mathrm{Al} / \mathrm{Cu}$ layers. IEEE JSelTop QuantElectron. 2017;23(2):4600408.
11. Tabassum R, Gupta BD. Performance analysis of bimetallic layer with zinc oxide for SPR-based fiber optic sensor. IEEE JLightwave Technol. 2015;33(22):4565-4571.

12. Kant R, Tabassum R, Gupta BD. Integrating nanohybrid membranes of reduced graphene oxide: chitosan: silica sol gel with fiber optic SPR for caffeine detection. Nanotechnology. 2017;28:195502.

13. Arjmand M, Saghafifar H, Alijanianzadeh M, et al. A sensitive taperedfiber optic biosensor for the label-free detection of organophosphate pesticides. Sens Actuators B. 2017;249:523-532.

14. Tabassum R, Gupta BD. Simultaneous estimation of vitamin $K_{1}$ and heparin with low limit of detection using cascaded channels fiber optic surface plasmon resonance. BiosensBioelectron. 2016;86:48-55. 